\title{
Assessment of spontaneous eye blink rate in online livestream video game players
}

\begin{abstract}
Introduction: The normal blink rate for humans is between 12 and 15 blinks per minute (bpm). Screen use has been associated with decreased blink rate and increased percentage of incomplete blinks, however, little is known about the effects of videogaming on blink rate. The purpose of this study was to assess the blink rate of online livestream video game players.

Methods: A total of 30 livestream video gamers across YouTube, Twitch, and Facebook Gaming were selected for this study. Video gamers were characterized by gender and whether or not they were wearing glasses. Blink rate was observed for 5 minutes and defined as a complete closure of the ocular surface. Descriptive statistics and linear regression were used to determine significance $(\mathrm{p}<0.05)$ between variables. All analyses were conducted using RStudio (version 1.3.1056; RStudio, Inc).

Results: The study population comprised of 30 video game players ( $50 \%$ men). The mean (standard deviation [SD]) blink rate of the total study population was 14.79 (8.79) bpm. Compared to male gamers, female gamers had a higher mean blink rate, however this was not statistically significant $(17.07$ vs. $12.51, \mathrm{p}=0.16)$. A total of $3(10 \%)$ video game players wore glasses. The difference in blink rate between video game players wearing and not wearing glasses was not statistically significant ( 14.07 vs $14.87, \mathrm{p}=0.88)$.

Conclusions: The average blink rate of select livestream video game players was observed to be within the range of normal spontaneous blink rates. The observed blink rate between male and female gamers did not differ. More research in controlled environments would help to elucidate the blink rate and patterns of video game players.
\end{abstract}

Volume I I Issue I - 202I

\section{Joana Andoh, Brian DeBroff}

Department of Ophthalmology and Visual Science, Yale School of Medicine, USA

Correspondence: Brian DeBroff, Department of Ophthalmology and Visual Science, Yale School of Medicine, New Haven, Connecticut, USA, Email brian.debroff@yale.edu

Received: March 04, 2021 | Published: March 15, 2021

\section{Introduction}

Spontaneous eye blinking is a hallmark of a healthy and normally functioning human. Occurring at a normal frequency of 12 to 15 times per minute, blinking helps to promote the homeostasis of the ocular surface. Ocular-related pathologies, such as dry eye disease (DED) and computer vision syndrome (CVS) have been related to dysregulation of the tear film and subsequent blinking. With the rise of electronic device usage and screen time, studies have started to investigate the effect of electronic displays on spontaneous eye blink rate (SEBR) and patterns. Electronic video displays and video games have been found to decrease SEBR in healthy individuals. However, other studies have noted no significant changes in SEBR but an increase in incomplete blinks. Despite these advances in better understanding SEBR and electronic displays, there is a paucity of literature that explored SEBR in livestream video game players. Livestream video game platforms, such as YouTube and Twitch, have gained popularity. Professional and amateur video game players continuously stream their gaming sessions for hours at time. The purpose of this study was to determine the SEBR in livestream video game players. Requiring concentration, like the activity reading, but requiring a video display, we hypothesize that livestream video game players will exhibit a blink rate lower than normal SEBRs. ${ }^{1-7}$

\section{Methods}

This study received exempt status from Institution Review Board of Yale University and abided by the tenets of the Declaration of Helsinki. Livestream video game players were queried using the online platforms YouTube, Twitch, and Facebook Gaming. Livestream videos were selected for observation if they were unedited, continuous, and had full view of the gamer's eyes during the observation period. SEBR was calculated by sampling blinks for 5-minutes and averaging the number of blinks to determine SEBR per minute. A blink was defined as a complete closure of the ocular surface such that the maximal palpebral fissure closed by at least $50 \%$. Demographic variables including gender and whether or not the gamer wore glasses were collected. Descriptive statistics and linear regression were used to determine significance $(\mathrm{p}<0.05)$ between variables. All analyses were conducted using RStudio (version 1.3.1056; RStudio, Inc).

\section{Results}

The study population comprised of 30 video game players $(50 \%$ women). A total of $3(10 \%)$ video gamers wore glasses. The mean (standard deviation [SD]) blink rate of the total study population was 14.79 (8.79) bpm. The 25th, 50th, and 75th percentiles of SEBR for the total study population were $8.2,12.1$, and 20.2 , respectively (Table 1). Compared to male gamers, female gamers had a higher mean blink rate, however this was not statistically significant (17.07 vs. 12.51, $\mathrm{p}=0.16$ ) (Figure 1). The difference in mean blink rate between video gamers wearing and not wearing glasses was not statistically significant (14.07 vs 14.87, $\mathrm{p}=0.88$ ) (Table 2).

Table I Spontaneous eye blink rate of male and female online livestream video game players

\begin{tabular}{llll}
\hline & Total & Male & Female \\
\hline Percentile & $\mathbf{n = 3 0}$ & $\mathbf{n}=\mathbf{~ I 5}$ & $\mathbf{n}=\mathbf{~ I 5}$ \\
\hline $0 \%$ & 3.4 & 3.4 & 7.6 \\
$25 \%$ & 8.2 & 7.2 & 10.9 \\
$50 \%$ & 12.1 & 10.6 & 17.8 \\
$75 \%$ & 20.2 & 13.1 & 22 \\
$100 \%$ & 42.2 & 42.2 & 31 \\
\hline
\end{tabular}




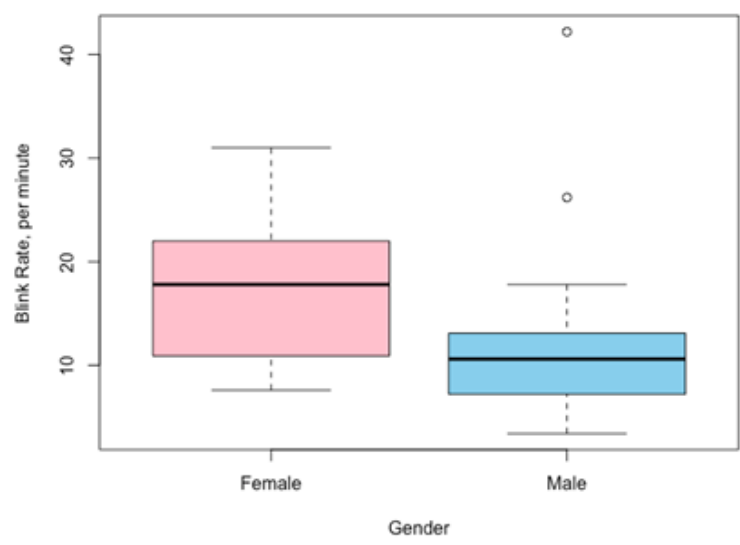

Figure I Boxplot of spontaneous eye blink rate of male and female video game players.

Table 2 Raw data: characteristics and blink measurements of male and female video game players

\begin{tabular}{|c|c|c|c|}
\hline Gender & Glasses & $\begin{array}{l}\text { Total Blinks } \\
\text { in } 5 \text { Minutes }\end{array}$ & Blinks/Minute \\
\hline $\mathrm{I}=$ Male & 0 & 58 & 11.6 \\
\hline I & 0 & 40 & 8 \\
\hline I & 0 & 68 & 13.6 \\
\hline I & 0 & 18 & 3.6 \\
\hline I & 0 & 39 & 7.8 \\
\hline I & 0 & 44 & 8.8 \\
\hline I & 0 & 63 & 12.6 \\
\hline I & 0 & 17 & 3.4 \\
\hline I & I & 55 & II \\
\hline I & 0 & 33 & 6.6 \\
\hline I & 0 & 211 & 42.2 \\
\hline I & 0 & 131 & 26.2 \\
\hline I & 0 & 19 & 3.8 \\
\hline I & 0 & 89 & 17.8 \\
\hline I & I & 53 & 10.6 \\
\hline $2=$ Female & 0 & 115 & 23 \\
\hline 2 & 0 & 112 & 22.4 \\
\hline 2 & 1 & 103 & 20.6 \\
\hline 2 & 0 & 38 & 7.6 \\
\hline 2 & 0 & 76 & 15.2 \\
\hline 2 & 0 & 39 & 7.8 \\
\hline 2 & 0 & 95 & 19 \\
\hline 2 & 0 & 54 & 10.8 \\
\hline 2 & 0 & 108 & 21.6 \\
\hline 2 & 0 & 55 & 11 \\
\hline 2 & 0 & 89 & 17.8 \\
\hline 2 & 0 & 155 & 31 \\
\hline 2 & 0 & 117 & 23.4 \\
\hline 2 & 0 & 77 & 15.4 \\
\hline 2 & 0 & 47 & 9.4 \\
\hline
\end{tabular}

\section{Discussion}

The mean SEBR (14.79 bpm) observed among study participants was found to be within the range of normal blink rates. However, SEBRs as low as $3.40 \mathrm{bpm}$ were observed among select video gamers. Studies examining the effects of visual displays on blink rate have found a reduced rate, as much as a $40 \%$ decrease when compared to rest. Previous studies of blink rate and video games have found results primarily based on the pace, size of display, visual dynamics, and cognitive requirements of the terminal task. A study in young participants observed a mean blink rate of $24.36 \mathrm{bpm}$ in baseline conditions, 12.44 in low dynamic visual displays, and 8.96 in high dynamic visual displays. ${ }^{6}$ Lee et al., ${ }^{12}$ also reported a progressive decrease in blinking with a mean rate of $8.27 \mathrm{bpm}$ after 1 hour and $9.51 \mathrm{bpm}$ after 4 hours of continuous gaming. A study of participants playing handheld video games (black-and-white visual display) observed a decrease in mean blink rate from $20.4 \mathrm{bpm}$ (baseline) to $5.6 \mathrm{bpm}$. Within this study, all SEBRs were recorded during the first two hours of livestream gaming, however, the sessions had varied game types and durations as long as six hours. Obtaining a baseline SEBR for each study participant, preferably at rest or primary gaze would help to determine whether the measured SEBR was relatively reduced due to video games. Overall, reduced blinking causes increased exposure of the ocular surface, putting stress on the cornea and contributing to symptoms of dry eye syndrome. ${ }^{10-13}$

In addition to reduced SEBRs, select video gamers in our study exhibited SEBRs as high as $42.2 \mathrm{bpm}$. One explanation could be that some streaming games involve loud noises or fast-moving stimuli projected toward the gamer. These types of stimuli may elicit an optic reflex blink reaction which serves an involuntary protective measure for the eye. Future studies will address different gaming stimuli (both sound and movement) to explore this issue further. Another explanation for increased blink rate among gamers may be related to a competitive advantage of staying alert and keeping the corneas maximally moistened for best visual performance. In fact, many gamers have been advised by coaches and eye care professionals to intentionally blink to maintain clear vision and reduce eye strain. This type of blink training has been shown to improve low SEBRs and impartial blinks associated with visual displays and video games. In such a manner, this study may have been biased by intentional blinking that is gaining popularity among video game players. A supplemental survey, examining the knowledge and conscious use of intentional blinking during gaming could help to better elucidate these findings. ${ }^{14-15}$

Findings of this study revealed no gender difference between male and female video gamers. Studies investigating gender and blink rate have yielded varied results. One study of 150 volunteers observed a higher blink rate among women when compared to men, however this was only while reading. Doughty et al. observed no difference in blink rate between young men and women during primary gaze activities. An additional study of gender and blink rate made note of women who were taking birth control pills. Women on birth control demonstrated a higher blink rate than women not on birth control and men, suggesting a mechanistic relationship between steroid hormones and spontaneous blinking. Given that DED is more prominent in menopausal and postmenopausal women, observing a higher blink rate in women may be related to the effects of estrogen and progesterone on the hydration of the ocular surface. This study, to our knowledge, is the first to investigate gender among video game players. Given the uncertainty still associated with gender and blinking, video games may be an appropriate medium to investigate such hypotheses. ${ }^{16-18}$

Another metric of exploration, which was not recorded in this study relates to incomplete blinks. Reduced blink amplitude can 
result in the impartial closure of the ocular surface, putting the inferior cornea at risk of damage. Portello et al. observed a positive correlation between the percentage of incomplete blinks and reported ocular symptoms. ${ }^{3}$ When compared to reading from a hard copy, subjects in multiple studies have shown an increase number of incomplete blinks when reading from a computer screen. ${ }^{7}$ Given that blinks in this study were detected via direct observation, it is possible that some of counted blinks could have technically been incomplete blinks. A possible solution to this study limitation could be the implementation of blink detection software. Aarts et al. ${ }^{20}$ utilized an eye tracker which recorded the reflection of light from the open eye. Thus, a complete blink would be defined as the absence of light reflection. Another form of blink detection software, developed by Monaro et al., ${ }^{21}$ has utilized an algorithm that identifies facial landmarks around the ocular adnexa and creates an Eye Aspect Ratio (EAR). Research related to facial paralysis and the tracking of healthy facial expressions has prompted the development of infrared-based blink detection, which can be attached to standard eyeglasses. Recently, blink detection software has attempted to refine algorithms to not only detect blinks, but also incomplete blinks. Fogelton et al has explored the use of recurrent neural networks $(\mathrm{RNN})$, a method of processing sequential data that accounts for differences in speed and duration. This class of machine learning has been previously applied in processing data such as speech recognition, DNA sequences, and text processing but has proven its utility in discriminating between complete and incomplete blinks. Overall, the recent emergence of alternative means for blink and incomplete blink detection offers additional opportunities for protocol development and follow-up studies. ${ }^{19-23}$

In conclusion, the findings of this study - in which the mean blink rate of video game players was within the range of normal blink rates - underscore the need to examine blink amplitude and rate. In the context of the COVID-19 pandemic and stay-at-home orders, the study of blinking and ocular pathologies has become more relevant. Increases in near work and digital screen time have put more individuals at risk of myopia and dry eye symptoms. Further investigation of blink patterns among video game players presents the opportunity to explore how dynamic visual displays affect symptoms of eye strain. Although this study did not include pediatric subjects, the emergence of asthenopia in children, dubbed Video Game Vision Syndrome, is also a point of interest. In conclusion, we hope this study can serve as a starting point for the exploration of blink patterns, video games, and the associations of ocular pathologies..$^{24-26}$

\section{Acknowledgments}

None.

\section{Conflicts of interest}

There are no conflicts of interest

\section{Funding}

None.

\section{References}

1. Stern JA, Walrath LC, Goldstein R. The endogenous eyeblink Psychophysiology. 1984;21(1):22-33.

2. Abusharha AA. Changes in blink rate and ocular symptoms during different reading tasks. Clin Optom (Auckl). 2017;9:133-138.

3. Portello JK, Rosenfield M, Chu CA. Blink Rate, Incomplete Blinks and Computer Vision Syndrome. Optometry and Vision Science. 2013;90(5):482-487.
4. Courtin R, Pereira B, Naughton G, et al. Prevalence of dry eye disease in visual display terminal workers: a systematic review and meta-analysis. BMJ Open. 2016;6(1):e009675.

5. Freudenthaler N, Neuf H, Kadner G, et al. Characteristics of spontaneous eyeblink activity during video display terminal use in healthy volunteers. Graefes Arch Clin Exp Ophthalmol. 2003;241(11):914-920.

6. Cardona G, García C, Serés C, et al. Blink Rate, Blink Amplitude, and Tear Film Integrity during Dynamic Visual Display Terminal Tasks. Current Eye Research. 2011;36(3):190-197.

7. Argilés M, Cardona G, Pérez-Cabré E, et al. Blink Rate and Incomplete Blinks in Six Different Controlled Hard-Copy and Electronic Reading Conditions. Invest Ophthalmol Vis Sci. 2015;56(11):6679-6685.

8. Kabiri NS, Brooks C, Comery T, et al. The Hawthorne Effect in Eyeblinking: Awareness that One's Blinks are Being Counted Alters Blink Behavior. Current Eye Research. 2020;0(0):1-5.

9. RStudio Team. Studio: Integrated Development for R. RStudio, PBC, Boston, MA; 2020.

10. Acosta MC, Gallar J, Belmonte C. The Influence of Eye Solutions on Blinking and Ocular Comfort at Rest and During Work at Video Display Terminals. Experimental Eye Research. 1999;68(6):663-669.

11. Yamada F. Frontal midline theta rhythm and eyeblinking activity during a VDT task and a video game: useful tools for psychophysiology in ergonomics. Ergonomics. 1998;41(5):678-688.

12. Lee J-W, Cho HG, Moon B-Y, et al. Effects of prolonged continuous computer gaming on physical and ocular symptoms and binocular vision functions in young healthy individuals. PeerJ. 2019;7.

13. Tsubota K, Miyake M, Matsumoto Y, et al. Visual protective sheet can increase blink rate while playing a hand-held video game. American Journal of Ophthalmology. 2002;133(5):704-705.

14. Downie LE, Craig JP. Tear film evaluation and management in soft contact lens wear: a systematic approach. Clin Exp Optom. 2017;100(5):438-458.

15. Murakami D, Blackie C, Korb D. Blinking exercises can be used to decrease partial blinking and improve gland function and symptoms in patients with evaporative dry eye. American Academy of Optometry. 2021.

16. Bentivoglio AR, Bressman SB, Cassetta E, et al. Analysis of blink rate patterns in normal subjects. Movement Disorders. 1997;12(6):1028-1034.

17. Doughty MJ. Further Assessment of Gender- and Blink Pattern-Related Differences in the Spontaneous Eyeblink Activity in Primary Gaze in Young Adult Humans. Optometry and Vision Science. 2002;79(7):439 447.

18. Peck T, Olsakovsky L, Aggarwal S. Dry Eye Syndrome in Menopause and Perimenopausal Age Group. J Midlife Health. 2017;8(2):51-54.

19. Chu CA, Rosenfield M, Portello JK. Blink Patterns: Reading from a Computer Screen versus Hard Copy. Optometry and Vision Science. 2014;91(3):297-302.

20. Aarts H, Bijleveld E, Custers R, et al. Positive priming and intentional binding: Eye-blink rate predicts reward information effects on the sense of agency. Social Neuroscience. 2012;7(1):105-112.

21. Monaro M, Capuozzo P, Ragucci F, et al. Using Blink Rate to Detect Deception: A Study to Validate an Automatic Blink Detector and a New Dataset of Videos from Liars and Truth-Tellers. In: Kurosu M, editor. Human-Computer Interaction. Human Values and Quality of Life. Lecture Notes in Computer Science. Springer International Publishing; 2020. p. 494-509.

22. Frigerio A, Hadlock TA, Murray EH, et al. Infrared- Based Blink Deteching Glasses For Facial Pacing: Towards a Bionic Blink. JAMA Facial Plast Surg. 2014;16(3):211-218.

23. Fogelton A, Benesova W. Eye blink completeness detection. Computer Vision and Image Understanding. 2018;176-177:78-85. 
24. Colley R, Bushnik T, Langlois K. Exercise and screen time during the COVID-19 pandemic. Health reports. 2020;31:3-11.

25. Mull A. Yes, the Pandemic Is Ruining Your Body. The Atlantic. 2021.
26. Rechichi C, De Mojà G, Aragona P. Video Game Vision Syndrome: A New Clinical Picture in Children? J Pediatr Ophthalmol Strabismus. 2017;54(6):346-355. 